\title{
Ab Initio Based Tight-Binding Hamiltonian for the Dissociation of Molecules at Surfaces
}

\author{
Axel Gross* and Matthias Scheffler \\ Fritz-Haber-Institut der Max-Planck-Gesellschaft, Faradayweg 4-6, D-14195 Berlin-Dahlem, Germany \\ Michael J. Mehl and Dimitrios A. Papaconstantopoulos \\ Complex Systems Theory Branch, Naval Research Laboratory, Washington, D.C. 20375-5345
}

(Received 29 July 1998)

\begin{abstract}
A tight-binding total-energy (TBTE) method has been developed to interpolate between firstprinciples results describing the dissociation of molecules at surfaces. The TBTE scheme requires only a relatively small number of $a b$ initio energies as input and gives a reliable global representation of the $a b$ initio potential energy surface to within $0.1 \mathrm{eV}$ accuracy compared to the $a b$ initio results. This approach will open the way to the $a b$ initio molecular dynamics description of reactions invoking many atoms and long time scales that are currently not accessible by first-principles methods. [S0031-9007(99)08464-1]

PACS numbers: 68.35.Ja, 82.20.Kh, 82.65.Jv
\end{abstract}

The theoretical study of reactions at surfaces is of strong current interest. In recent years it has become possible to map out the potential energy surfaces (PES) describing reactions at surfaces in great detail by $a b$ initio methods [15]. However, in order to obtain reaction rates that can be compared with experiment it is neccessary to calculate the dynamics of the nuclei which move according to $a b$ initio forces. It is by now well known that the complexity of the high-dimensional PES is significant and even in the simplest case the phase space is twelve dimensional. In fact, the number of crucial coordinates of the configuration space might easily exceed 12 or 20 or even more, in particular if surface degrees of freedom are involved in the reaction [6]. The determination of reaction probabilities for such processes requires the calculation of $10^{3}-10^{6}$ trajectories to ensure reliable statistics [7]. We note that the proper treatment of the statistics is indeed as important as a good-quality electronic structure calculation for determining the PES. Since the $a b$ initio total-energy calculations require a considerable computational effort, it is therefore necessary to have a method that gives a reliable interpolation between the calculated $a b$ initio points. In this paper we will show that a tight-binding (TB) method [8-10] is a good candidate for fulfilling these requirements.

There are several approaches to fit potential energy surfaces. All methods have advantages and disadvantages. It is therefore fair to say that the issue of the best fitting scheme for a particular problem is not settled yet. A common interpolation scheme is to fit the $a b$ initio data to some analytical representation, which has, for example, successfully been used for the ab initio description of the hydrogen dissociation on metal surfaces using a six-dimensional PES [7,11-13]. Once a reliable analytical fit is found, it is computationally inexpensive to calculate the potential gradients at arbitrary configuration which is needed, e.g., to perform molecular dynamics simulations of reactions. However, if more degrees of freedom are involved in a particular reaction, then it is very cumbersome to find an appropriate analytical form. As an alternative, the interpolation of $a b$ initio points by a neural network has been proposed [14-16]. Neural networks require no assumptions about the functional form of the underlying problem, but a relatively large number of $a b$ initio input points is needed for an accurate description. Recently, a genetic programming scheme which searches for both the best functional form and the best set of parameters has been investigated [17]. This method has so far been used only for threedimensional potentials so that a proof of its applicability for higher-dimensional problems is still missing.

The common denominator of all the methods mentioned so far is that they allow a fast determination of the fitted PES at arbitrary configurations. However, these methods can provide a reliable description of potential energy surfaces of multidimensional reactions only if based on a sufficiently high number of $a b$ initio points. Assuming that for each degree of freedom at least three points are necessary, we estimate that in six dimensions $10^{3}$ and in twelve $10^{6}$ ab initio total energy calculations are required. Consequently, an intermediate step is needed. In this paper we use a nonorthogonal tight-binding total-energy (TBTE) method for fitting an ab initio PES of the dissociation of molecules at surfaces. To our knowledge, TB methods have hardly been used for the treatment of reactions at surfaces [18]. Hence it has not been clear whether a TB scheme is capable of describing the complex multidimensional PES of such a reaction which is caused by the variety of covalent interactions, charge transfer, and bond breaking and making at various geometries and orientations with respect to the surface. In this paper we show that our TB method is not only able to accurately reproduce an ab initio PES, but also needs only a moderate number of input total energies for a reliable global description of the PES. This is due to the fact that the quantum mechanical nature of bonding is taken into account properly [18], and that the 
parameters of the TBTE method, the Slater-Koster integrals [19], have a physical meaning.

The computational effort to determine total energies with a TBTE method is larger by 2 to 3 orders of magnitude compared to an analytical representation since it requires the diagonalization of matrices. Still it is faster by at least 2 to 3 orders of magnitude than $a b$ initio total-energy methods (since the different methods scale differently with, e.g., the number of atoms considered, these estimates are only approximate and depend on the actual system). Because of its accuracy and speed, our TB method is well suited to extend the application of ab initio molecular dynamics to systems which are currently not accessible by first-principles methods.

The NRL TBTE method we use in this study has been described in detail before [8-10]. Therefore we will only briefly summarize its essentials and the modifications we have introduced for the particular problem of fitting an $a b$ initio PES of molecular dissociation at surfaces. Unlike other tight-binding methods [18,20-22], this TBTE scheme does not include a pair-potential term. Instead, the method takes advantage of the fact that the total energy of a system is independent of the choice of zero of the potential. In the TBTE method this shift, which depends on the structure and volume, is determined by the requirement that the total energy of the system be just the sum of the shifted eigenvalues $\epsilon_{i}^{\prime}$ :

$$
E_{\mathrm{tot}}=\sum_{\mathrm{occ}} \epsilon_{i}^{\prime}
$$

The $\epsilon_{i}^{\prime}$ are the eigenvalues of the generalized Schrödinger equation

$$
\left(\hat{\mathbf{H}}-\epsilon_{i}^{\prime} \hat{\mathbf{S}}\right) \psi_{i}=0,
$$

where $\hat{\mathbf{H}}$ and $\hat{\mathbf{S}}$ are the Hamiltonian and overlap matrices, respectively, in an atomic basis representation $\left\{\phi_{\alpha}\right\}$. The Hamiltonian and overlap parameters are assumed to have the two-center, nonorthogonal Slater-Koster form [19]. Their dependence on the distance $r$ between two atoms is parametrized according to

$$
P_{i}=\left(\sum_{k=0}^{k_{\max }^{(i)}} a_{k}^{(i)} r^{k}\right) \exp \left(-\gamma_{i}^{2} r\right) f_{i}(r),
$$

where

$$
f_{i}(r)=\frac{1}{1+\exp \left[\left(r-r_{i}^{0}\right) / l\right]}
$$

is a cutoff function. The index $i$ is a multicomponent index that labels the interacting orbitals. The method also contains environment-dependent on-site terms that account for the effects of the local neighborhood on each atom [8-10].

In the first applications of the NRL method the TB parameters were chosen to simultaneously fit the band structures and total energies from a set of density-functional theory (DFT) calculations in the local density approxima- tion (LDA) for transition and noble metal crystals [8,9]. These TB parameters are available via the WWW [23]. This method is able to predict elastic constants, vacancy formation, and surface energies in very good agreement with both DFT-LDA calculations and experiment. In the meantime the method has been extended to the complicated $\alpha \mathrm{Mn}$ structure [24], group-III elements [25], and binary compounds, e.g., $\mathrm{PdH}$ [26].

In this paper we apply the binary version of the NRL tight-binding scheme to the calculation of the PES of molecular dissociation on surfaces. The particular system we have chosen is the dissociation of hydrogen on the $\operatorname{Pd}(100)$ surface. This is a well-studied system, both experimentally [27-29] and theoretically $[3,7,11,30]$. The PES of $\mathrm{H}_{2} / \mathrm{Pd}(100)$ has been determined in great detail [3] using density-functional theory (DFT) together with the generalized gradient approximation (GGA) [31]. An analytical representation of this six-dimensional ab initio PES has been used for quantum and classical dynamics calculations in which all hydrogen degrees of freedom were treated dynamically $[7,11]$. We have used the more than 250 calculated points of the $a b$ initio PES as a data base to find out whether the TBTE method is capable of reproducing the PES.

In the tight-binding scheme $\mathrm{Pd}$ is described by nine orbitals $(s, p$, and $d)$ while for hydrogen we use the $1 s$ orbital. In fitting the PES we have used only total energies, i.e., no band structures. Still for a $\mathrm{H}_{2}$ molecule far away from the $\mathrm{Pd}$ surface, i.e., in a situation where the $\mathrm{H}_{2}$ and the Pd surface are not coupled, the vacuum level $\epsilon_{\infty}$ for both subsystems (hydrogen molecule and Pd surface) should be aligned. This leads to the relation

$$
\epsilon_{\infty}=\epsilon_{\mathrm{F}}+\Phi=\epsilon_{\sigma_{g}}+I .
$$

Here $\epsilon_{\mathrm{F}}$ is the Fermi energy of $\mathrm{Pd}, \Phi$ is the work function of the $\operatorname{Pd}(100)$ surface, $\epsilon_{\sigma_{g}}$ is the energy of the $\sigma_{g}$ level of the hydrogen molecule, and $I$ is the ionization energy of the hydrogen molecule. In our fitting scheme we vary only the hydrogen on-site terms and the $\mathrm{H}-\mathrm{H}$ and $\mathrm{Pd}-\mathrm{H}$ Hamiltonian and overlap parameters. The Pd on-site terms and Pd-Pd parameters are kept fixed; they have been determined in Refs. $[9,23]$. However, while the polynomial appearing in the functional form of the Pd-Pd parameters in Eq. (3) is expanded only up to the first order term, i.e., $k_{\max }^{\text {Pd-Pd }}=1$, we realized that for the $\mathrm{H}-\mathrm{H}$ and $\mathrm{Pd}-\mathrm{H}$ parameters this expansion is not flexible enough to accurately reproduce the $\mathrm{H}_{2} / \mathrm{Pd}(100) a b$ initio PES. This is due to the fact that in order to describe the process of bond breaking and bond making at surfaces, the Slater-Koster parameters have to be appropriately determined for a wide range of distances. For that reason we expand the polynomial in Eq. (3) for the $\mathrm{H}-\mathrm{H}$ and $\mathrm{Pd}-\mathrm{H}$ parameters up to fourth order. In addition, we have changed the cutoff length $r_{i}^{0}$, which is $14 a_{\mathrm{B}}$ for the Pd-Pd parameters ( $a_{\mathrm{B}}$ denotes the Bohr radius), to $8.5 a_{\mathrm{B}}$ for the $\mathrm{H}-\mathrm{H}$ and $\mathrm{H}-\mathrm{Pd}$ parameters. 
In total we have 53 parameters to be adjusted in order to reproduce the $a b$ initio PES. This adjustment is done by a nonlinear least-squares fit [9]. We always adjust the TB parameters to simultaneously reproduce the $\mathrm{H}-\mathrm{H}$ vibration in the gas phase and the $\mathrm{H}_{2} / \mathrm{Pd}(100)$ PES. This ensures that we obtain the right asymptotic behavior for the $\mathrm{H}_{2}$ molecule far away from the surface. The $\mathrm{H}_{2} / \mathrm{Pd}(100)$ PES is determined within a $c(2 \times 2)$ unit cell, as in the DFTGGA calculations [3].

An important issue for the fitting procedure is the fact that not all configurations are equally important for the determination of reaction probabilities. For example, the dissociation dynamics at lower kinetic energies depend crucially on whether there is a low minimum barrier of height $0.1 \mathrm{eV}$ hindering the dissociation in the entrance channel or not [32]. On the other hand, a deviation of the potential by $0.2 \mathrm{eV}$ in a region where the potential is already elevated might have almost no influence on the reaction probabilities. As a consequence, the root-meansquared (rms) error, which is usually used as an indicator for the quality of a fit, is of only limited value. The nonlinear least-square fitting routine implemented in the tight-binding code allows a weighting factor to be associated with every input energy. We use these weighting factors in order to ensure a most accurate reproduction of the most relevant region of the PES. For activated systems with a nonvanishing minimum energy barrier $E_{b}$ this will be the region where the potential energy differs from $E_{b}$ by less than, say, $0.5 \mathrm{eV}$. For a nonactivated system like $\mathrm{H}_{2} / \mathrm{Pd}(100)$ a criterion for the importance of a particular region is not that obvious to establish. We associated the points with potential energies close to zero and points along the minimum energy path in Fig. 1(a) with weights which were up to 10 times larger than the weights associated with the rest of the points. The points that have been used to obtain the fit are marked by the dots. In total we needed only 55 input points for fitting the TB-PES.

Figure 2 illustrates that our TB scheme also reproduces the $a b$ initio energies for a fixed lateral $\mathrm{H}_{2}$ center of mass above the fourfold hollow site, but different molecular orientations. In Figs. 2(a) and 2(b) the molecular axis is parallel to the surface, but the azimuthal orientation is different. Molecules dissociating towards the bridge site between two Pd atoms do not encounter any energy barrier (Fig. 2a), while molecules dissociating towards Pd atoms (Fig. 2b) eventually are repelled by the Pd atoms. Figure $2 \mathrm{c}$ finally demonstrates that hydrogen molecules oriented perpendicularly to the surface cannot dissociate.

The contour plots shown in Figs. 1 and 2 should be compared with the $a b$ initio input data in Ref. [3]. The comparison reveals that the fit reproduces the minimum energy paths and also the general shape of the elbow plots very well. The overall rms error, including all the $a b$ initio values that were not fitted, is only $0.1 \mathrm{eV}$, a value that is usually considered to be within the accuracy of the $a b$ initio total energies.
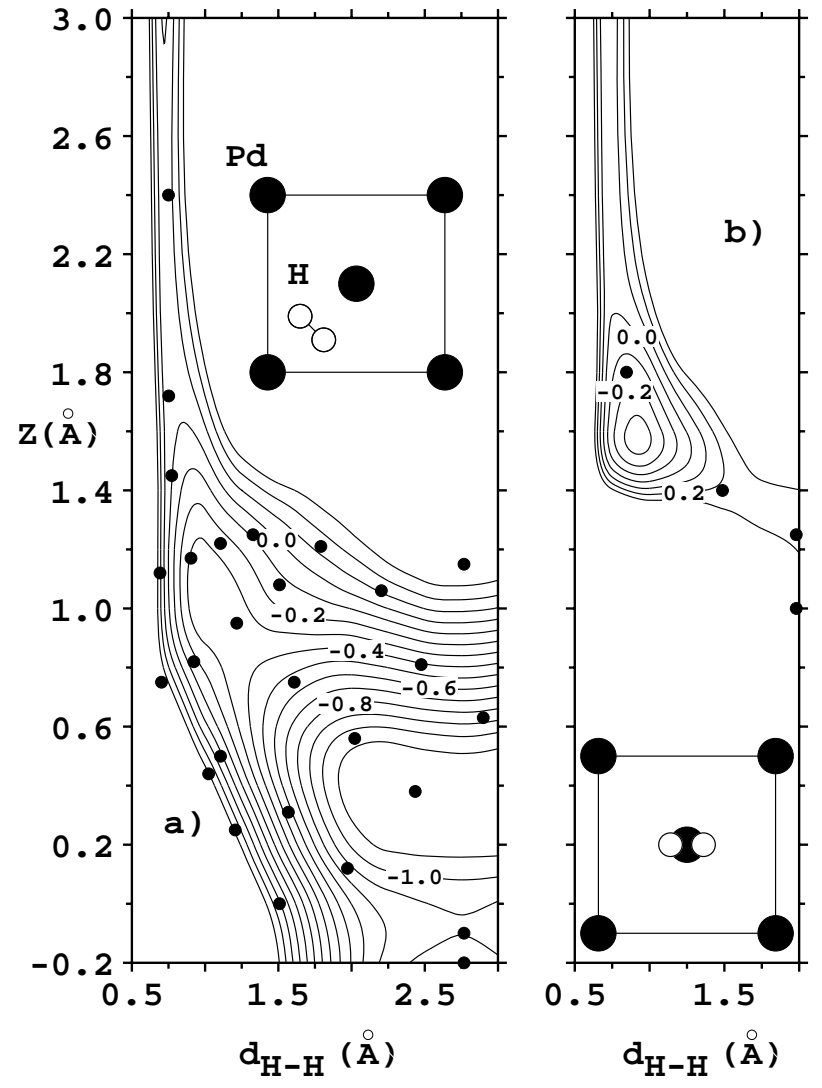

FIG. 1. Contour plots of the TB-PES along two twodimensional cuts through the six-dimensional coordinate space of $\mathrm{H}_{2} / \mathrm{Pd}(100)$. The coordinates in the figure are the $\mathrm{H}_{2}$ centerof-mass distance from the surface $Z$ and the $\mathrm{H}-\mathrm{H}$ interatomic distance $d_{\mathrm{H}-\mathrm{H}}$. The lateral $\mathrm{H}_{2}$ center-of-mass coordinates in the surface unit cell and the orientation of the molecular axis, i.e., the coordinates $X, Y, \theta$, and $\phi$ are kept fixed for each $2 \mathrm{D}$ cut and depicted in the insets. The molecular axis is kept parallel to the surface; (a) corresponds to the dissociation at the bridge site, (b) to dissociation at the top site. The dots denote the points that have been used to obtain the fit. Energies are in $\mathrm{eV}$ per $\mathrm{H}_{2}$ molecule. The contour spacing is $0.1 \mathrm{eV}$.

The agreement between $a b$ initio input energies and the TB energies can be further improved if the Pd-Pd parameters are also varied (the rms error drops to $0.07 \mathrm{eV}$ ). However, purposely we have presented only the results for the adjusted $\mathrm{H}-\mathrm{H}$ and $\mathrm{H}-\mathrm{Pd}$ parameters. The Pd parameters are already fitted to $a b$ initio calculations for Pd bulk. They already reproduce bulk properties such as elastic constants and surface properties, e.g., surface energies for different low-index surfaces [9]. In previous dynamical simulations of the $\mathrm{H}_{2}$ dissociation on $\mathrm{Pd}(100)$ the substrate has been kept rigid [7,11]. In order to assess the influence of the surface motion on the dissociation the PES in the six molecular degrees of freedom has to be determined as a function of the coordinates of the substrate atoms. This necessitates the calculation of total energies for an enormous number of different configurations, and thus represents an ideal application of the $a b$ initio TB method. 


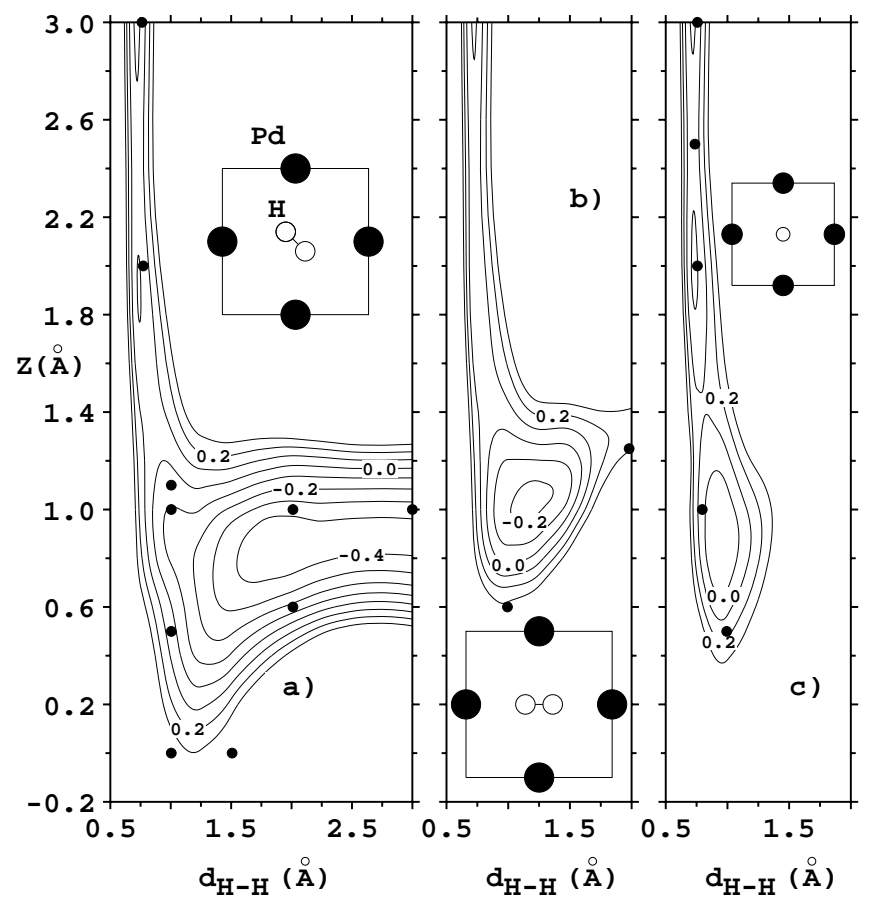

FIG. 2. Contour plots of the TB-PES along three twodimensional cuts through the six-dimensional coordinate space of $\mathrm{H}_{2} / \mathrm{Pd}(100)$ for the $\mathrm{H}_{2}$ center of mass above the fourfold hollow site. The representation is analogous to Fig. 1. The insets illustrate the orientation of the molecule. (a) Molecule parallel to the surface dissociating towards the bridge sites between two Pd atoms; (b) molecule parallel to the surface dissociating towards the Pd atoms; (c) molecule perpendicular to the surface.

Either one can use the TB method directly to perform tightbinding molecular dynamics simulations, or one can use the information obtained by the TB total energies to adjust the parameters of a flexible interpolation scheme like, e.g., a neural network [16] which allows a fast evaluation of total energies, but needs a large number of input points for adjusting the parameters. We plan to perform calculations along these lines in the future.

In conclusion, we have shown that a tight-binding totalenergy scheme is able to reproduce the $a b$ initio PES of the dissociation of molecules at surface. Since in the tightbinding method the quantum mechanical nature of bonding is taken into account, only a limited set of $a b$ initio input points is needed in order to give a reliable representation of the global PES. Thus the $a b$ initio TB scheme is well suited to extend the results of $a b$ initio calculations. Possible applications are tight-binding molecular dynamics calculations involving many atoms and trajectories or the extension of ab initio calculations for fitting schemes that need many input points.

A. G. would like to thank the Naval Research Laboratory for its hospitality during a stay where this work was initiated. Work at NRL is sponsored by the U.S. Office of Naval Research.
*New address: Physik-Department T30, Technische Universität München, D-85747 Garching, Germany.

[1] B. Hammer, M. Scheffler, K. W. Jacobsen, and J. K. Nørskov, Phys. Rev. Lett. 73, 1400 (1994).

[2] J. A. White, D. M. Bird, M. C. Payne, and I. Stich, Phys. Rev. Lett. 73, 1404 (1994).

[3] S. Wilke and M. Scheffler, Phys. Rev. B 53, 4926 (1996).

[4] A. Eichler, G. Kresse, and J. Hafner, Phys. Rev. Lett. 77, 1119 (1996).

[5] C. Stampfl and M. Scheffler, Phys. Rev. Lett. 78, 1500 (1997).

[6] A. Gross, M. Bockstedte, and M. Scheffler, Phys. Rev. Lett. 79, 701 (1997).

[7] A. Gross and M. Scheffler, Phys. Rev. B 57, 2493 (1998).

[8] R. E. Cohen, M. J. Mehl, and D. A. Papaconstantopoulos, Phys. Rev. B 50, 14694 (1994).

[9] M. J. Mehl and D. A. Papaconstantopoulos, Phys. Rev. B 54, 4519 (1996).

[10] M.J. Mehl and D. A. Papaconstantopoulos, in Topics in Computational Materials Science, edited by C. Y. Fong, (World Scientific, Singapore, 1998), Ch. 5.

[11] A. Gross, S. Wilke, and M. Scheffler, Phys. Rev. Lett. 75, 2718 (1995).

[12] G. Wiesenekker, G. J. Kroes, and E. J. Baerends, J. Chem. Phys. 104, 7344 (1996).

[13] C. M. Wei, A. Gross, and M. Scheffler, Phys. Rev. B 57, 15572 (1998).

[14] T. B. Blank, S. D. Brown, A. W. Calhoun, and D. J. Doren, J. Chem. Phys. 103, 4129 (1995).

[15] K. T. No, B. H. Chang, S. Y. Kim, M. S. Jhon, and H. A. Scheraga, Chem. Phys. Lett. 271, 152 (1997).

[16] S. Lorenz, A. Gross, and M. Scheffler, APS Bull. 43, 235 (1998).

[17] D. E. Makarov and H. Metiu, J. Chem. Phys. 108, 590 (1998).

[18] G. M. Goringe, D. R. Bowler, and E. Hernández, Rep. Prog. Phys. 60, 1447 (1997).

[19] J. C. Slater and G. F. Koster, Phys. Rev. 94, 1498 (1954).

[20] M. M. Sigalas and D. A. Papaconstantopoulos, Phys. Rev. B 49, 1574 (1994).

[21] M. Menon, J. Connolly, N. Lathiotakis, and A. Andriotis, Phys. Rev. B 50, 8903 (1994).

[22] A. E. Carlsson, Phys. Rev. B 44, 6590 (1991).

[23] WWW address: http://cst-www.nrl.navy.mil/bind

[24] M. J. Mehl and D. A. Papaconstantopoulos, Europhys. Lett. 31, 537 (1995).

[25] S. H. Yang, M.J. Mehl, and D. A. Papaconstantopoulos, Phys. Rev. B 57, R2013 (1998).

[26] D. A. Papaconstantopoulos and M. J. Mehl, Mater. Res. Soc. Symp. Proc. 408, 31 (1996).

[27] G. Comsa, R. David, and B.-J. Schumacher, Surf. Sci. 95, L210 (1980).

[28] K. D. Rendulic, G. Anger, and A. Winkler, Surf. Sci. 208, 404 (1989).

[29] L. Schröter, H. Zacharias, and R. David, Phys. Rev. Lett. 62, 571 (1989); Surf. Sci. 258, 259 (1991).

[30] A. Eichler, G. Kresse, and J. Hafner, Surf. Sci. 397, 116 (1998).

[31] J. P. Perdew et al., Phys. Rev. B 46, 6671 (1992).

[32] A. Gross, C. M. Wei, and M. Scheffler, Surf. Sci. 416, L1095 (1998). 